\title{
Procesos de automatización cognitiva en alumnado con altas capacidades intelectuales
}

\author{
Juan Montero-Linares, José I. Navarro-Guzmán* y Manuel Aguilar-Villagrán
}

Departamento de Psicología. Universidad de Cádiz.

\begin{abstract}
Resumen: Proponemos desde el enfoque del procesamiento de la información, el estudio de características cognitivas que faciliten la identificación de las altas capacidades. A tal fin, elaboramos una tarea de habilidad de segmentación silábica que, a partir del modelo propuesto por Baddeley sobre la memoria de trabajo y la distinción entre procesos automáticos y controlados, nos permitiese comprobar las diferencias en cuanto a la capacidad de automatización de la información y si los indicadores propuestos en la misma, tenían suficiente poder clasificatorio o predictivo en la identificación de la alta capacidad intelectual. Participaron 480 niños y niñas de primero a cuarto de Educación Primaria. Se clasificaron, en función de su CI, en dos grupos: alumnado de altas capacidades y alumnado de capacidades medias. Se les administró una batería de pruebas entre las que se encontraba el test de habilidad de segmentación silábica, lo que permitió realizar su estandarización. Los resultados mostraron que los alumnos con CI superior a 130 fueron capaces de automatizar la segmentación de forma más eficiente que los de inteligencia media. La medición de los procesos automáticos a través del test de habilidad de segmentación silábica, permitió diferenciar a los participantes de altas capacidades de los que no lo eran.

Palabras claves: Altas capacidades; identificación; memoria de trabajo; procesamiento de la información; procesos automáticos; procesos controlados.
\end{abstract}

\section{Introducción}

La inteligencia se ha considerado como la variable imprescindible en la definición y evaluación de los alumnos con altas capacidades (Renzulli, 1998; Sternberg, 1999). Sin embargo, no se nos ocultan las dificultades para definir con precisión y de modo universal qué es la inteligencia y, en consecuencia, responder con claridad a la pregunta qué son las altas capacidades. La aparición de los modelos cognitivos de procesamiento de la información han traído nuevas formas de identificación hacia enfoques más cualitativos y orientados al estudio de otros componentes, tales como los modos de percibir, recordar y pensar, o las formas distintas de aprehender, almacenar y utilizar la información que tienen los individuos (Prieto y Hervás, 2001; Sanz de Acedo y Sanz de Acedo, 2008). Las dificultades para detectar, identificar y evaluar al alumno de altas capacidades, se plantean tanto desde el punto de vista de su conceptualización como desde las técnicas e instrumentos empleados (Carman, 2006; Corbalán y Limiñana, 2010). Resulta, pues, conveniente conocer qué teoría subyace en la concepción de las altas capacidades, por las repercusiones que tiene en la selección de instrumentos de identificación en la práctica educativa.

Es objeto de debate si los niños con altas capacidades automatizan la información más eficazmente que los de inteligencia media y si ello, les confiere una ventaja inicial que les

* Dirección para correspondencia [Correspondence address]: José I. Navarro. Departamento de Psicología. Universidad de Cádiz. 11510 Puerto Real, Cádiz, (España). E-mail: jose.navarro@uca.es
Title: Automatic cognitive processing in gifted and non gifted students. Abstract: The traditional gifted children assessment model has several insufficiencies. It is based on IQ data as a main definition of giftedness. However this paper proposes other perspective from the processing information theory. We studied other cognitive variables that could be used for gifted children's assessment. A syllabic segmentation skills test was designed after the working memory's Baddeley and automatic and controlled processes models by Shneider y Shiffrin. The syllabic segmentation skills test allows learning more about the routine information processing, and it is able to prove its predictive capacity identifying gifted students. A total of 480 primary school children from 6 to 9 years old participated in this study. After IQ were assessed with the Wechsler Intelligence Scale for Children (WISC), students were distributed in two different groups (gifted and non gifted). All participants were also assessed by the syllabic segmentation skills test. Different measurements for syllabic segmentation skills test's standardization were collected. Results shown that gifted children had significant higher scores on syllabic segmentation than non gifted. This suggests that children with a IQ over 130 were able to make routine information processing faster than lower performers, and the syllabic segmentation skills test was able to discriminate gifted and non gifted students.

Key words: Gifted; high intellectual abilities; working memory; information processing; automatic process; controlled process.

facilite el manejo de la información en procesos de pensamiento más complejos (Lépine, Barrouillet, y Camos, 2005). El éxito de la realización de una tarea depende del paso del procesamiento controlado al procesamiento automatizado (Manzanero, 2006), ya que se liberan recursos de atención que pueden utilizarse para otros procesos de mayor dificultad, y porque la automatización del conocimiento permite una representación más compleja del problema. De este modo, son diversas las explicaciones actuales sobre las diferencias individuales y de desarrollo que subrayan la importancia del papel que juegan algunos procesos cognitivos a la hora de determinar la conducta inteligente compleja. Las teorías del procesamiento de la información conceden importancia al hecho de que los sujetos difieren en la rapidez y automatización con que realizan tareas simples (Proctor y Vu, 2006). Así, los sujetos que tardan en identificar la información entrante o que necesitan centrar toda su atención en la tarea, puede que carezcan de los recursos atencionales necesarios para realizar actividades complejas de nivel superior que necesiten componentes integradores. Por ejemplo, en una tarea de lectura, un alumno con un nivel lector deficiente, puede ocupar todos sus recursos de atención en los procesos de decodificación de las letras o palabras y, por ello, tener serias dificultades para el acceso a la comprensión del texto (Finton, 2008). Tareas de este tipo evidencian su relación con las capacidades atencionales, con la velocidad de procesamiento, con la automatización de la información y con un uso eficaz de la memoria de trabajo (Kavanagh y Mohan, 2008; Baddeley, 2010).

En relación con lo anterior, la investigación sugiere que las personas pueden variar mucho en su capacidad de me- 
moria operativa, la cantidad de información que se puede mantener accesible, y que estas diferencias predicen el nivel de inteligencia general, evaluado mediante pruebas estandarizadas para obtener el CI (Conway, Kane y Engle, 2003). Partiendo de la actual concepción de la memoria de trabajo (Baddeley, 2010), las evidencias disponibles apuntan que las diferencias individuales en factor " $g$ ” podrían ser explicadas por los conceptos cognitivos de capacidad y de velocidad asociados a este tipo de memoria (Colom, Rebollo, Palacios, Espinosa, y Kyllonen, 2004). En concreto, las diferencias en inteligencia se relacionarían con el componente principal de la memoria de trabajo, el ejecutivo central.

En la actualidad, la memoria de trabajo, se entiende como el conjunto de items que, en un momento dado, se encuentran activos y están siendo utilizados voluntariamente por el sujeto. La concepción actual es más bien de tipo funcional. Baddeley (2010) propone que la memoria de trabajo (MT) está formada por al menos tres subsistemas (ejecutivo central, bucle fonológico y agenda visoespacial) organizados de forma jerárquica entre sí y que funcionan en estrecha colaboración. Dentro de este modelo correspondería al ejecutivo central el control voluntario del procesamiento de la información, facilitando o inhibiendo el pensamiento o la acción en curso. Sería un sistema de naturaleza atencional que ejerce el control voluntario y la toma de decisiones, estrechamente relacionado con la experiencia consciente.

Por otra parte, la diferenciación entre procesos automáticos y controlados (Schneider y Shiffrin, 1977; Hower, Vandorpe y Beckers, 2005) y cómo, con la práctica y experiencia suficientes, una tarea controlada puede convertirse en automática, nos proporcionan otra oportunidad para el estudio de las diferencias individuales. En este sentido, existen diferencias acerca del funcionamiento cognitivo, entre los alumnos de altas capacidades y los alumnos de capacidades medias, en lo referente a procesos psicológicos básicos tales como: atención, memoria de trabajo y los procesos de automatización de la información (Bailey, Dunlosky y Kane, 2008; Colom y Flores-Mendoza, 2001). De este modo, a partir del modelo propuesto por Baddeley (2010) sobre la memoria de trabajo y la distinción entre procesos automáticos y controlados, tratamos de calibrar las diferencias en cuanto a la capacidad de automatización de la información, entre niños/as de altas capacidades y de capacidad intelectual media, y si los indicadores propuestos en la medida de la segmentación silábica (segmentar las sílabas, invertirlas y sintetizarlas), tendrían suficiente poder clasificatorio o predictivo en su identificación.

Las implicaciones de este modelo en la elaboración de nuestra tarea de habilidades de segmentación silábica, se centra en el funcionamiento del supervisor atencional (ejecutivo central), como mecanismo de control voluntario de las operaciones realizadas en la memoria a corto plazo (repetición continua de palabras en orden inverso), y en la utilización del bucle fonológico como almacén subsidiario de la información verbal (retención de la palabra) y del sistema de control articulatorio (en nuestro caso: articulación no subvocal de las palabras o ítems de la tarea). En este sentido, resultaría lógico pensar que el alumnado con altas capacidades tuviese un mayor desarrollo en estos procesos psicolingüísticos, favoreciendo un aprendizaje precoz de la lectura, a diferencia de los que siguen una progresión normal o que presentan dificultades para su iniciación, dada su inmadurez para analizar el lenguaje oral. Pretendíamos diseñar una prueba que cumpliese como requisitos básicos los siguientes: (a) Fácil de aplicar, de corregir y cuya administración fuese breve. (b) Capaz de discriminar entre alumnado de altas capacidades y de capacidad intelectual media. Finalmente, (c) capaz de activar procesos psicológicos básicos tales como atención, memoria de trabajo, velocidad de procesamiento y capacidad para automatizar la información.

Asumiendo estos planteamientos y conscientes de la existencia de un gran número de tests que tratan de medir estos procesos, pensamos que la mayoría de ellos van dirigidos a la medida de la atención sostenida, a la valoración de la amplitud de la memoria a corto plazo (visual o auditiva) y de la memoria de trabajo, mediante el incremento sucesivo de la dificultad de la tarea. Sin embargo, nuestro interés fundamental se centraba en el estudio del ejecutivo central como mecanismo de control del flujo o procesamiento de la información y de la capacidad de los sujetos para convertir un proceso controlado en automático. Además, el tipo de tarea a realizar debería guardar cierta relación con el aprendizaje de la lectura y con la mayor o menor capacidad de los sujetos para automatizar la información.

Por todo lo anterior, decidimos seleccionar una tarea de procesamiento fonológico (repetición de palabras bisílabas en orden inverso) que cumpliese los requisitos siguientes: a) Presentar un elevado grado de similitud fonológica para incrementar la dificultad de la tarea y en consecuencia la demanda atencional para discriminar entre palabras muy parecidas. Baddley (1966) contrastó estas predicciones presentando listas de palabras con parecido fonológico y con parecido semántico, concluyendo que las listas con similaridad fonológica se recuerdan peor, mientras que no ocurre lo mismo con las listas con similaridad semántica que se comportan igual que sus listas de control. b) Escasa demanda de amplitud de la memoria de trabajo. Dado que difícilmente podemos operar mentalmente con más de 6 ó 7 elementos independientes a la vez, elegimos palabras bisílabas que permitiesen operar con facilidad sin comprometer dicha capacidad de retención y minimizando el efecto de longitud de la palabra sometido a prueba por Baddeley, Thomson y Buchanan (1975). c) Relacionada con la conciencia fonológica o predictiva para el aprendizaje de la lectura. Para evaluar la conciencia fonológica se suelen utilizar tareas de segmentación de palabras en sílabas. Las capacidades de segmentación están relacionadas directa y teóricamente con el aprendizaje de la lectura, encontrándose diferencias significativas en conciencia fonológica (silábica, entre otras) entre buenos y malos lectores (Jiménez y O’shanahan, 2008). d) Con significación semántica tanto en orden directo como inverso, facilitando su aprendizaje y automatización mediante procesos 
de memoria asociativa. Y que se tratara de palabras con un índice bajo de dificultad articulatoria.

El objetivo por tanto de nuestro trabajo fue desarrollar una tarea capaz de evaluar las habilidades de segmentación silábica y diferenciar el potencial de los participantes para automatizar la información de modo eficiente, así como obtener una función predictiva sobre la identificación de alumnado con altas capacidades intelectuales. Consideramos que aquellos sujetos con mayor inteligencia automatizan más rápidamente la información, liberando recursos cognitivos para enfrentarse a tareas más complejas.

\section{Método}

\section{Participantes}

La muestra inicial estuvo formada por un total de 480 participantes. Cincuenta y dos por 100 alumnos y 48\% alumnas de Educación Primaria, procedentes de 4 centros educativos públicos y de 2 privados-concertados de una localidad de 120.000 habitantes, procedentes de 3 zonas educativas con carácterísticas socioculturales diferenciadas: zona centro o de nivel sociocultural medio-alto; zona mediaemergente de nivel medio-medio, y zona periférica o de educación compensatoria de nivel medio-bajo. El alumnado de $1^{\circ}$ de educación primaria fue un total de 87 (46 niños y 41 niñas); los de $2^{\circ}$ de educación primaria fueron 136 (69 niños y 67 niñas); en $3^{\circ}$ fueron 135 (73 niños y 62 niñas); y en $4^{\circ}$ un total de 122 (61 niños y 61 niñas).

\section{Instrumentos}

Para la selección de los grupos de altas capacidades intelectuales (AC) y capacidades medias $(\mathrm{CM})$ y para realizar los estudios de fiabilidad, validez y tipificación del Test de Habilidades de Segmentación Silábica (THSS), se utilizaron las siguientes pruebas:

Test de Habilidades de Segmentación Silábica (THSS). Se trata de una prueba diseñada para evaluar la capacidad de los participantes para convertir un proceso controlado en automático. La tarea a realizar, consistía en pedirle a cada participante individualmente que repitiese en orden inverso diez palabras (cosa, lave, toga, pata, sabe, llama, vena, lapa, paso, copa), anotándose el tiempo empleado en cada repetición del listado. De este modo, durante los 7 minutos que dura la prueba se repetiría un número determinado de veces (las que el niño realice en el tiempo asignado). Se contabilizaban, pues, los tiempos parciales de cada repetición del listado y además el número total de palabras correctamente emitidas. A partir de los resultados se obtuvieron tres indicadores:

1. Índice principal (AS): atención sostenida o memoria de trabajo: La motivación y la tenacidad del sujeto en la realización de una tarea de ejecución continua, donde las pérdidas atencionales son penalizadas con el incremento del tiempo de respuesta, reflejarían su capacidad de atención sostenida y el funcionamiento de la memoria de trabajo (ejecutivo central) para operar de manera continuada con la información retenida. La puntuación directa viene dada por el número total de palabras repetidas correctamente durante los 7 minutos que dura la prueba.

2. Índice cualitativo (PAI): potencial de automatización intrasujeto: Definido como la capacidad para mejorar el tiempo empleado en la repetición del listado de palabras. Viene expresado por la proporción entre el tiempo empleado la primera vez y el más bajo conseguido. Este índice no permite comparar a los sujetos pero sí contabilizar su aptitud o potencial para automatizar la tarea. Por ello, se trata de un indicador acerca de su propia realización. Su cálculo vendría dado por la expresión: $\mathrm{PAI}=\mathrm{T} 1 / \mathrm{Tb}$. Donde: $\mathrm{PAI}=$ Potencial de Automatización; T1 = Tiempo empleado la primera vez en la repetición del listado; $\mathrm{Tb}=$ Tiempo más bajo conseguido en la repetición del listado. Se trataría de comparar la mejora conseguida en relación con la primera ejecución de la tarea. Los valores obtenidos serán menores o mayores que 1 , situándose el cero de la escala en la posición central (valor 1). Ello ocurriría cuando ambos valores sean iguales $(\mathrm{T} 1=\mathrm{Tb}) . \mathrm{Si} \mathrm{Tb}<$ T1, el potencial será $>1$. Si Tb $>$ T1 el índice será $<1$. Por ejemplo: un participante que hubiese obtenido las puntuaciones siguientes: $\mathrm{T} 1=150$ segundos; $\mathrm{Tb}=86$ segundos; obtendría un PAI $=150 / 86=1.74$. Este resultado supone una mejora de su rendimiento en un $74 \%$, consiguiendo cierto nivel de automatización de la información. Por el contrario, si hubiese obtenido un PAI $=.62$ supondría una disminución de un $38 \%$, lo cual nos podría sugerir una escasa capacidad para la automatización o la aparición de síntomas de cansancio o de fatiga.

3. Índice derivado (PAIS): potencial de automatización intersujetos. Con el indicador anterior (PAI) sólo podemos saber la capacidad del sujeto o potencial para mejorar su propia puntuación, pero no nos serviría para establecer comparaciones con los demás, ya que se trata de una medida intrasujeto. Sin embargo, con este nuevo indicador PAIS podemos conocer además de su posición relativa en la curva normal, si pertenece o no al grupo de sujetos que automatizan la tarea. Para obtener la puntuación de este indicador procedimos a tipificar las puntuaciones $\mathrm{T} 1$ y $\mathrm{Tb}$ ( $\mathrm{T} 1 \mathrm{z}$ y $\mathrm{Tbz}$, respectivamente), a partir de las cuales y mediante la aplicación de la fórmula PAIS $=(\mathrm{T} 1 z+\mathrm{Tbz}) / 2$, calculamos la puntuación típica obtenida por el sujeto. A partir de este valor podemos clasificar a los sujetos en dos grandes grupos: sujetos que automatizan la tarea y sujetos que no la automatizan.

Test de matrices progresivas, escalas general y en color (Raven, 1994), que ofrece índices de fiabilidad entre .80 y 93. Se trata de un test no verbal, donde el sujeto describe las piezas que faltan de una serie de láminas impresas. Se pretende que el sujeto utilice habilidades perceptuales, de 
observación y razonamiento analógico para deducir el estímulo que falta en la matriz. Se le pide al sujeto que analice la serie que se le presenta y que siguiendo la secuencia horizontal y vertical, escoja uno de los ocho trazos el que encaje perfectamente en ambos sentidos, tanto en el horizontal como en el vertical. Prueba de evaluación de la inteligencia de valor contrastado y de amplia utilización por profesionales de la psicología y pedagogía, que fue administrada de forma colectiva en pequeños grupos siguiendo los criterios de edad y condiciones que señalan los manuales de realización de la escala general y en color. Se justificó su uso en esta investigación por razones funcionales, dada la economía en tiempo y esfuerzo que supone su administración, así como su capacidad discriminante teniendo en cuenta sus índices de validez y fiabilidad.

Escala de inteligencia de Wechsler para niños (WISC-IV; Wechsler, 2005), con índice de fiabilidad que variaba entre .86 a .95 , y coeficientes de validez elevados (.65 a .89). De administración individual, ofrece información sobre la capacidad intelectual general del niño (CI total) y sobre su funcionamiento en las principales áreas específicas de la inteligencia (compresión verbal, razonamiento perceptivo, memoria de trabajo y velocidad de procesamiento). La escala se compone de 15 tests, 10 principales y 5 optativos.

Test de intersección de figuras (FIT) (Pascual Leone y Johnson, 2001). El diseño de la prueba está basado en la teoría de los operadores constructivos de Pascual-Leone (1978), que propone la existencia de un mecanismo de atención mental, memoria de trabajo o procesador inmediato llamado Operador $M$. El poder máximo de $M$ viene dado por $e$ (capacidad $M$, desarrollada en el periodo sensoriomotor) y el parámetro $k$ cuyo valor aumenta de uno a siete, entre los 3-4 años y los 15-16, indicando el número de esquemas que el sujeto puede manejar de manera simultánea. La finalidad de esta prueba es medir el parámetro $k$ equivalente al número de tareas bien resueltas por los participantes. En el FIT, cada reactivo presenta un grupo de diferentes figuras geométricas con diverso grado de superposición. El participante debe hacer una señal con el lápiz en el lugar de la intersección de todas las figuras geométricas. El tiempo de administración del FIT es de 20 a 30 minutos. La fiabilidad de la prueba está establecida en .90 usando el coeficiente alpha de Cronbach.

Tareas de atención sostenida en la infancia (CSAT) (Servera y Llabrés, 2004). Se trata de una prueba computerizada de ejecución continua para la medida de la capacidad de atención sostenida en la infancia y fundamentada en teoría de detección de señales. En esta prueba la pantalla del ordenador muestra una serie de números, de 0 a 9 , cada 500 milisegundos, y esos números permanecen en pantalla durante 250 milisegundos. El niño debe fijarse bien y cada vez que vea un 6 y a continuación se presente un 3 , debe pulsar la barra espaciadora del teclado, pero no debe pulsar la barra ante cualquier otra combinación de números. El número total de estímulos que presenta la pantalla es de 600, de entre los cuales la combinación 6-3 se da en un 30\% de los casos. La duración total de la prueba, sin contabilizar el período de ensayo, es de 7 minutos y 30 segundos. De aplicación individual y dirigida a niños desde los 6-7 hasta los 10-11 años. Presenta baremos por grupos de edad coincidentes con los niveles escolares de $1^{\circ}$ a $4^{\circ}$ de Educación Primaria. Ofrece índices de fiabilidad basados en el procedimiento test-retest (valores entre .73 y .77) y coeficientes de correlación entre bloques de la prueba (valores entre .80 para aciertos y omisiones y alrededor de .85 para los tiempos de reacción).

Test de los cinco dígitos (FDT) (Sedó, 2007). Esta prueba permite describir la velocidad de procesamiento, fluidez verbal, la atención sostenida y la eficiencia en la alternancia entre procesos mentales automáticos y controlados. Consta de cuatro partes de aplicación independiente, en las que se presentan series de 50 casillas que contienen de 1 a 5 dígitos (partes I, III y IV) o estrellas (parte II) cada una, organizados en patrones similares a los de las figuras de dominó o las cartas de juego. En la parte I se pide al niño/a que lea lo más rápido posible, de izquierda a derecha, en filas, el dígito que contiene cada casilla. En la parte II se le pide que cuente, también en filas y tan rápido como pueda, cuántas estrellas contienen cada casilla. En la parte III se le pide que cuente el número de dígitos que contiene cada casilla, produciendo un efecto de interferencia, ya que las casillas presentan grupos de dígitos que no se corresponden con su valor aritmético (por ejemplo, en una casilla con el número 2 cinco veces repetido, la respuesta correcta sería 5 y no 2). Esta sección equivale a la condición de interferencia del test de Stroop. Por último, en la parte IV se le pide que cuente, tal y como hizo en la parte III, o lea, tal y como hizo en la parte I, en función de que el recuadro de la casilla sea normal (contar, $80 \%$ de los estímulos), o de doble grosor (leer, $20 \%$ de los estímulos). Las partes III y IV son sensibles al funcionamiento de procesos ejecutivos de atención selectiva y supresión de respuestas automáticas. De aplicación individual y colectiva a niños, adolescentes y adultos, con una duración de 5 minutos. Posee un elevado grado de fiabilidad (entre .80 y .94). En cuanto a su estructura interna y sus relaciones con otras pruebas similares, consigue un grado de validez elevado (entre .57 y .91) dependiendo de los distintos tipos de tareas considerados.

Batería de evaluación de procesos lectores-Revisada (PROLECR). Para la evaluación de la decodificación lectora se han utilizado dos subtests del test PROLEC-R de Cuetos, Rodríguez, Ruano, y Arribas (2007). Se administraron los subtests de lectura de palabras y pseudopalabras que requieren la correcta identificación de 40 palabras y 40 pseudopalabras. El alfa de Cronbach para el total del test es .79.

Calificaciones Escolares, extraídas de las actas de evaluación de final de curso, en las áreas de: Lenguaje, Matemáticas y Conocimiento del Medio.

\section{Procedimiento}

Los objetivos del trabajo fueron llevar a cabo los estudios de la fiabilidad y validez del THSS. Tras esto, plantemos 
las hipótesis que los índices de atención sostenida (AS), potencial de automatización inter sujetos (PAIS) y potencial de automatización intra sujetos (PAI), nos permitirían diferenciar entre alumnos de altas capacidades y de capacidades medias y que además podían predecir el status de clasificación de cada sujeto en ambos grupos. La investigación se realizó en dos fases bien diferenciados aunque su ejecución en el tiempo no fue necesariamente longitudinal. La primera, consistente en la detección e identificación de alumnos de altas capacidades (AC) y de capacidades medias (CM), y la segunda en la validación empírica del THSS.

Los criterios establecidos, en la primera fase, para la formación de ambos grupos de participantes de nuestro estudio (AC y CM) fueron los siguientes:

1. Para el grupo de altas capacidades (AC): Obtener un cociente intelectual igual o superior a 120 en el test de Raven escala general. Rendimiento notable o sobresaliente en las materias instrumentales de Lenguaje, Matemáticas y Conocimiento del Medio. Obtener un cociente intelectual total igual o superior a 130 en la escala de inteligencia de Weschsler para niños (WISC-IV). También se incluyeron aquellos casos que además contaban con un in- forme previo de alta capacidad intelectual, emitido por el Equipo de Orientación Educativa.

2. Para el grupo de capacidades medias (CM): Que sus profesores los identificasen como sujetos de capacidad intelectual normal. Rendimiento suficiente o notable en las materias instrumentales de Lenguaje, Matemáticas y Conocimiento del Medio. Obtener un cociente intelectual total superior a 85 e inferior a 116 en la aplicación de la escala de inteligencia de Weschsler para niños (WISCIV). No recibir ningún tipo de refuerzo educativo en el centro escolar.

Tras la aplicación de los criterios anteriormente señalados, se seleccionó una muestra final de 50 participantes (Tabla 1). De ellos, 25 fueron clasificados como de altas capacidades y 25 de capacidades medias. En cuanto al género, 30 eran niños $(60 \%)$ y 20 niñas $(40 \%)$. El rango de edad variaba entre los 6.12 y 10.85 años con una media de 8.38 años y una desviación típica de 1.10 años. En la población inicial evaluada de $N=480$ hubo más de 25 participantes que cumplían los criterios del grupo de CM. Sin embargo, se seleccionaron al azar sólo 25 para lograr un equilibrio estadísticamente válido entre los dos grupos de estudio (AC y $\mathrm{CM}$ ).

Tabla 1. Distribución de alumnos y alumnas con altas capacidades intelectuales (AC) y de capacidades medias (CM), por niveles educativos, edad (media y desviación típica, DT) y género.

\begin{tabular}{|c|c|c|c|c|c|c|c|c|c|}
\hline \multirow[b]{4}{*}{ Grupos } & \multicolumn{8}{|c|}{ Niveles educativos } & \multirow{4}{*}{ Total } \\
\hline & \multicolumn{2}{|r|}{$1^{o}$} & \multicolumn{2}{|r|}{$2^{\circ}$} & \multicolumn{2}{|r|}{$3^{\circ}$} & \multicolumn{2}{|r|}{$4^{\circ}$} & \\
\hline & Edad media & $6.63, D T=.38$ & Edad media & $7.71, D T=.29$ & Edad media & $8.83, D T=.31$ & Edad media & $9.77, D T=.30$ & \\
\hline & Niños & Niñas & Niños & Niñas & Niños & Niñas & Niños & Niñas & \\
\hline $\begin{array}{l}\mathrm{AC} \\
\mathrm{CI} \geq 130\end{array}$ & 1 & 2 & 5 & 3 & 4 & 4 & 5 & 1 & 25 \\
\hline $\begin{array}{l}\mathrm{CM} \\
85 \leq \mathrm{CI} \leq 117\end{array}$ & 1 & 2 & 5 & 3 & 4 & 4 & 5 & 1 & 25 \\
\hline Total & 2 & 4 & 10 & 6 & 8 & 8 & 10 & 2 & 50 \\
\hline
\end{tabular}

En la segunda fase, para la comprobación de hipótesis y los estudios de la fiabilidad y validez del THSS se procedió del modo siguiente:

1. Aplicación individual del THSS a la totalidad de la muestra de partida $(N=480)$ con una duración aproximada de 10 minutos por participante.

2. Repetición de la aplicación del THSS a una submuestra de 85 participantes para el cálculo de la fiabilidad por el método test-retest.

3. Estudio de su validez concurrente, mediante el análisis de sus relaciones con las pruebas aplicadas a diferentes muestras: Raven escala color y PROLEC-R $(n=23)$; Raven escala general y calificaciones escolares en Lenguaje, Matemáticas y Conocimiento del Medio $(n=262)$; WISC-IV, FIT, CSAT y FDT $(n=50)$.

Todas las evaluaciones fueron realizadas por uno de los autores de este trabajo en buenas condiciones de aplicación y siguiendo las instrucciones de uso de las diferentes tareas. Los participantes lo fueron de manera voluntaria, se contó con el consentimiento informado de sus padres o tutores y de los centros escolares donde se desarrolló el estudio. Para el análisis de datos utilizamos el paquete estadístico SPSS 15.0 y la hoja de cálculo Excel 97.

\section{Resultados}

\section{Cálculo de la validez y fiabilidad del THSS}

Se ha estudiado la validez concurrente del test de habilidades de segmentación silábica, mediante el procedimiento correlacional con otras pruebas que miden constructos similares (FIT, CSAT, FDT), con medidas del rendimiento escolar (calificaciones de las asignaturas de Lenguaje y Matemáticas) y de la inteligencia (puntuaciones en los tests Raven y WISC-IV) acerca de su valor predictivo. A este respecto, se encontraron correlaciones significativas con el rendimiento escolar (.46 a .80), con la inteligencia (.41 a .723) y con los constructos similares (.41 a .67). Por otro lado, el índice de frabilidad total fue de $r=.89$, calculado por el método testretest, para una muestra de 85 casos (Tabla 2) y niveles de escolarización de $1^{\circ}$ a $4^{\circ}$ de Educación Primaria. 
Tabla 2. Coeficiente de fiabilidad test-retest obtenida en una selección de sujetos de la muestra de baremación del test de habilidades de segmentación silábica en la atención sostenida y en el potencial de automatización intersujetos.

\begin{tabular}{lrrrrr}
\hline $\begin{array}{l}\text { Curso } \\
\text { escolar }\end{array}$ & $n$ & $\begin{array}{l}\text { Atención } \\
\text { sostenida }\end{array}$ & $\begin{array}{l}\text { E.T.M } \\
\text { P.D. }\end{array}$ & $\begin{array}{l}\text { Potencial de automatiza- } \\
\text { ción intersujetos }\end{array}$ & $\begin{array}{l}\text { E.T.M } \\
\text { P.T.Z }\end{array}$ \\
\hline $1^{\circ}$ & 20 & .90 & 7 & .84 & .30 \\
$2^{\circ}$ & 23 & .77 & 16 & .71 & .26 \\
$3^{\circ}$ & 20 & .88 & 13 & .61 & .25 \\
$4^{\circ}$ & 22 & .86 & 17 & .90 & .23 \\
Total & 85 & .89 & 17 & .68 & .26 \\
\hline
\end{tabular}

E.T.M.: Errores típicos de medida. P.D.:Puntuaciones directas. P.T.Z: Puntuaciones tipicas $Z$.

Nuestras hipótesis de partida hacian referencia a que los índices de atención sostenida (AS), potencial de automatización inter sujetos (PAIS) y potencial de automatización intra sujetos (PAI), nos permitirían diferenciar entre alumnos de altas capacidades y de capacidades medias y que además podían predecir el status de clasificación de cada sujeto, incluido como de altas capacidades o de capacidades medias.

Para la primera cuestión, los resultados nos indicaron, en efecto, que sí existieron diferencias significativas tanto en lo referido al índice principal AS como al índice derivado PAIS entre los dos grupos. Los valores de estas diferencias fueron: AS: $t(48)=5.66, p<.001)$ y PAIS: $t(48)=5.79, p<.001)$. En este sentido, podemos concluir que los participantes de AC demostraron mayor capacidad de atención sostenida para retener y operar de manera continuada en tareas de segmentación de palabras (índice AS). De igual modo, su mayor velocidad para procesar la información les permitió automatizar la tarea consiguiendo tiempos más bajos (esto es, mejores resultados) que sus compañeros de capacidades medias (índice PAIS). En cuanto a la capacidad para mejorar la propia realización o estilo personal de funcionamiento (potencial de automatización intrasujeto, PAI), no se detectaron diferencias significativas entre ambos grupos (PAI: $t(48)=$ $.85 ; p$ n.s.).

Para la verificación del valor predictivo de los distintos índices utilizamos un análisis lineal discriminante por pasos, donde las variables se van incorporando a la función discriminante una a una. De esta manera, es posible, por un lado, construir una función utilizando únicamente aquellas variables que realmente son útiles para la clasificación y, por otro, evaluar la contribución individual de cada variable al modelo discriminante. Podemos efectuar así una clasificación de los mismos casos utilizados para obtener la función, y la posibilidad de hacerlo para casos futuros de los que, conociendo su puntuación en las variables independientes, se desconozca el grupo al que pertenecen. Previamente, se comprobaron los prerrequisitos básicos establecidos para este modelo respecto a las variables dependiente e independientes, así como al supuesto de homocedasticidad (Pérez, 2005).

Como puede apreciarse en la Tabla 3, existen diferencias significativas para los índices AS y PAIS, por lo que podemos rechazar la hipótesis nula que establece que los grupos no difieren entre si. No ocurre lo mismo con el tercer indicador (PAI), que quedará descartado para su inclusión en el análisis discriminante al no cumplir uno de los requisitos del mismo. En la prueba $\mathrm{M}$ de Box y su transformación en un estadístico $F$, se obtuvo un resultado, $F(1,6912)=.027, p<$ .870 , que no nos permite rechazar la hipótesis nula de que las matrices de covarianzas poblacionales sean iguales.

Tabla 3. Análisis discriminante descriptivo. Significación de las diferencias entre ambos grupos: altas capacidades y capacidades medias para los índices de atención sostenida (AS); potencial de automatización intersujetos (PAIS); y potencial de automatización intrasujeto (PAI).

\begin{tabular}{lccc}
\hline & $\lambda$ de Wilks & $F(1,48)$ & $p$ \\
\hline AS & .47 & 54.03 & .001 \\
PAIS & .59 & 33.04 & .001 \\
PAI & .98 & .72 & .400 \\
\hline
\end{tabular}

La Tabla 4 (autovalores y $\lambda$ de Wilks) nos muestran el porcentaje de varianza explicada por la función 1, que es del $100 \%$ de la representación muestral, así como una correlación moderadamente alta (.728) entre la función discriminante y la pertenencia al grupo. El poder de discriminación de la función es altamente significativa, como indica su autovalor (1.12), contrastado con la probabilidad de $\chi^{2}(1, N=$ $50)=35.82, p<.001$.

Tabla 4. Autovalores, $\lambda$ de Wilks y Chi-cuadrado de las diferencias entre los grupos de altas capacidades y capacidades medias.

\begin{tabular}{lcccc}
\hline Función & Autovalor & $\begin{array}{c}\% \text { de } \\
\text { varianza }\end{array}$ & $\begin{array}{c}\% \\
\text { acumulado }\end{array}$ & $\begin{array}{c}\text { Correlación } \\
\text { canónica }\end{array}$ \\
\hline 1 & 1.12 & 100 & 100 & .728 \\
$\begin{array}{l}\text { Contraste de } \\
\text { funciones }\end{array}$ & $\lambda$ de Wilks & $\chi^{2}$ & $g l$ & $p$ \\
1 & .47 & 35.82 & 1 & .001
\end{tabular}

La matriz de estructura nos muestra el peso predictivo de cada una de las variables, siendo el índice AS, con un peso de 1 , el que muestra mayor valor de predicción, el índice potencial de automatización intersujetos (PAIS) alcanzó un valor .761, y el potencial de automatización intrasujeto (PAI) de .095. La única variable seleccionada en el análisis fue el índice AS, cuyo valor del $\lambda$ de Wilks (.470) aunque no es muy pequeño, la probabilidad asociada de $\lambda$ Wilks y el estadístico $F(1,48)=54.039, p<.001$, confirmaron la significatividad de dos ejes discriminantes, con lo que su capacidad explicativa será buena, esto es, separa bien los dos grupos. Para describir la función discriminante se usaron los coeficientes estandarizados o canónicos: D1 $=1 *$ índice AS; D1 = $8.618+.077 *$ índice AS

Finalmente y para dar sentido a este análisis, hemos elaborado una tabla de contingencia (Tabla 5) para comparar los resultados de la clasificación de los grupos establecidos en el estudio -altas capacidades, capacidades medias- con los que se han pronosticado en el análisis discriminante realizado. Los resultados nos muestran el alto valor predictivo de la variable AS, ya que de los 25 participantes clasificados como de altas capacidades en el estudio, 21 fueron identificados en ese estatus mediante la prueba THSS. Es decir, clasificó correctamente al 84\% de los casos, frente al 80\% de los clasificados en el grupo de capacidades medias. 
Tabla 5. Tabla de contingencia para grupos de pertenencia pronosticado y real de los participantes de altas capacidades (AC) y de capacidad intelectual media (CM).

\begin{tabular}{|c|c|c|c|c|c|}
\hline & & & \multicolumn{2}{|c|}{$\begin{array}{l}\text { Grupo de pertenencia } \\
\text { pronosticado }\end{array}$} & \multirow{2}{*}{$\begin{array}{c}\text { Grupo de } \\
\text { pertenencia real } \\
\text { Total } \\
\text { participantes }\end{array}$} \\
\hline & & & $\mathrm{AC}$ & $\mathrm{CM}$ & \\
\hline \multirow{4}{*}{ Grupos } & & Recuento & 21 & 4 & 25 \\
\hline & & $\%$ grupos & $84 \%$ & $16 \%$ & $100 \%$ \\
\hline & \multirow{2}{*}{$\mathrm{CM}$} & Recuento & 5 & 20 & 25 \\
\hline & & $\%$ grupos & $20 \%$ & $80 \%$ & $100 \%$ \\
\hline \multirow{2}{*}{ Total } & & Recuento & 26 & 24 & 50 \\
\hline & & $\%$ grupos & $52 \%$ & $48 \%$ & $100 \%$ \\
\hline
\end{tabular}

$X^{2}(1, N=50)=20.51, p<.001$

\section{Discusión}

Pretendíamos conocer qué grado de automatización alcanzaban los sujetos participantes de nuestro estudio en la realización de una tarea de segmentación silábica que se repetía de manera continuada, y si el nivel de automatización conseguido establecía diferencias de ejecución entre dos grupos de alumnos de inteligencia superior y de inteligencia media. Pudimos verificar que ambos grupos discrepaban significativamente tanto en su capacidad de atención sostenida/memoria de trabajo (AS) como en la mayor velocidad para automatizar la tarea (PAIS); no así en cuanto al modo o estilo personal de funcionamiento (PAI). Estos datos nos hacen pensar en una mayor eficacia de los alumnos de altas capacidades frente al grupo de participantes de capacidades medias en su capacidad de automatización. Resultados que concuerdan con los obtenidos por otros autores (Jausovec, 2000; Hoppe, y Stojanovic, 2009), donde atribuyen el éxito de la realización de una tarea al paso del procesamiento controlado al procesamiento automatizado, realizando diferentes evaluaciones neurofisiológicas.

Junto a esta mayor capacidad para automatizar la tarea, también pudimos constatar un funcionamiento más eficaz de la memoria de trabajo, y más específicamente, en relación con el funcionamiento del supervisor atencional (ejecutivo central), como mecanismo de control voluntario de las operaciones realizadas en la memoria a corto plazo, en la utilización del bucle fonológico y del sistema de control articulatorio en la dirección referida por Baddeley (2003) y Alsina y Sáiz (2003). De manera análoga, otros estudios ponen de relieve la importancia de las relaciones entre la habilidad del ejecutivo central y el rendimiento aritmético, de modo que los niños que tienen menos disponibilidad de recursos de memoria tienen también un menor rendimiento en tareas de cálculo (Navarro, Aguilar, Marchena, Ruiz, Menacho, y Van Luit, 2011). Son muchas las explicaciones actuales sobre las diferencias individuales y de desarrollo, recogidas en la literatura y que subrayan el papel que juegan algunos procesos cognitivos a la hora de determinar la conducta inteligente compleja, y que algunos autores relacionan con las altas capacidades intelectuales (Colom y Flores-Mendoza, 2001; Kane y Engle, 2002; Badeley, 2003).

Dado el papel central que se atribuye a la memoria de trabajo en casi toda la actividad cognitiva (Lépine, Barrouillet, y Camos, 2005; Oberauer, Süb, Schulze, Wilhelm, y Wittmann, 2000) y dado sus límites, tanto en su capacidad como en el tiempo de operar, así como en el control del procesamiento actualizado de los códigos que se van activando, es lógico pensar que su funcionamiento establezca limitaciones y diferencias individuales sobre los procedimientos de orden superior como los cognitivos, los del lenguaje o especialmente en el inicio de los aprendizajes instrumentales de la lectura y el cálculo (Navarro, Aguilar, Alcalde, Ruiz, Marchena y Menacho, 2011; Ortiz et al., 2008).

La evidencia empírica que presentan nuestros resultados insisten en la memoria de trabajo y la capacidad de automatización, como procesos psicológicos optimizadores del buen funcionamiento cognitivo, y en consecuencia como prerrequisitos necesarios, aunque no suficientes para el alto funcionamiento intelectual. Más concretamente, la medición de estos procesos a través de la tarea de habilidades de segmentación silábica (THSS), que ha resultado tener valores de fiabilidad y validez estadísticamente significativos, permite discriminar con cierta precisión al alumnado de altas capacidades. Ello nos induce a pensar en la necesidad de elaborar protocolos de evaluación diagnóstica que superen el enfoque clásico e incluyan otros componentes o características asociadas a las altas capacidades. En este sentido, desde la práctica educativa, podrían plantearse programas que persigan como objetivo la detección e identificación tempranas, tanto de los alumnos con posible alta capacidad intelectual como de aquellos que puedan presentar un elevado riesgo de fracaso escolar, asociados a insuficiente estimulación o disfunciones de procesos psicológicos básicos de tipo atencional o relacionados con la memoria de trabajo. De este modo, se podrían poner en marcha estrategias de intervención psicopedagógica, a edades tempranas, para este sector de la población tales como: a) Programas de aceleración (adelanto de curso, compactación curricular...), enriquecimiento curricular (dirigido al contenido, proceso, producto o ambiente de aprendizaje) o la adopción de medidas de carácter organizativas (a nivel del aula ordinaria, aula de apoyo, agrupamientos flexibles...); y b) Programas de estimulación cognitiva de procesos psicológicos básicos (atención, memoria de trabajo, conciencia fonológica...) para aquellos que desde sus inicios presentan dificultades en sus aprendizajes, ya sean debidas a causas de origen sociocultural o de carácter personal y que muy probablemente desembocarían en un bajo rendimiento académico o fracaso escolar.

Agradecimientos.- Este trabajo fue parcialmente financiado por el proyecto de la Junta de Andalucía P09-HUM4918 y el proyecto EDU2011-22747 del plan nacional I+D+i. 


\section{Referencias}

Alsina, A. y Saíz, D. (2003). Un análisis comparativo del papel del bucle fonológico versus agenda visoespacial en el cálculo en niños de 7-8 años. Psicothema, 15, 241-246.

Baddeley, A. (1966). Short-term memory for word sequences as a function of acoustic, semantic and formal similarity. Quarteley Journal of Experimental Psychology, 18, 362-365.

Baddeley, A. (2003). Working memory and language: an overview. Journal of Communication Disorders. 36, 189-208. doi:10.1016/S0021-9924(03)00019-

Baddeley, A. (2010). Memoria de trabajo. En A. Baddley, M. W. Eysenck, y M. C. Anderson (Eds.), Memoria (pp. 63-91). Madrid: Alianza.

Baddeley, A., Thomsom, N. y Buchanan, M. (1975). Word lenght and the structure of short-term memory. Journal of Verbal Learning and Behavior, 14, 575-589.

Bailey, H., Dunlosky, J., \& Kane, M. (2008). Why does working memory span predict complex cognition? Testing the strategy affordance hypothesis. Memory y Cognition, 36, 1383-1390. doi:10.3758/MC.36.8.1383.

Carman, C.A. (2006). Relationships among traditional and modern constructs used in identifying giftdness. Roeper Review, 28, 111-112.

Colom, R. y Flores-Mendoza, C. (2001). Inteligencia y memoria de trabajo: la relación entre factor $g$, complejidad cognitiva y capacidad de procesamiento. Teoría e Pesquisa, 17, 37-47.

Colom, R., Rebollo, I., Palacios A., Espinosa, M.J., \& Kyllonen, P. C. (2004). Working memory is (almost) perfectly predicted by $g$. Intelligence, 32, 277-296. doi: 10.1016/j.intell.2003.12.002,

Conway A. R., Kane M. J. \& Engle R. W. (2003). Working memory capacity and its relation to general intelligence. Trends in Cognitive Sciences, 7, 547552. doi:10.1016/j.tics.2003.10.005

Corbalán, J. y Limiñana, R. M. (2010). El genio en una botella. El test CREA, las preguntas y la creatividad. Introducción al monográfico "El test CREA, inteligencia creativa". Anales de Psicología, 26, 197-205.

Cuetos, F., Rodríguez, B., Ruano, E. y Arribas, D. (2007). PROLEC-R. Bateria de evaluación de procesos lectores. Revisada. Madrid: TEA.

Finton, D. J. (2008). When Do Differences Matter? On-Line Feature Extraction Through Cognitive Economy. Recuperado de http://arxiv.org/abs/cs/0404032v1.

Hoppe, Ch., y Stojanovic, E. (2009). Giftedness and the brain. The Psychologist, 22, 498-501.

Hower, J., Vandorpe, S. y Beckers, T. (2005). On the role of controlled cognitive processes in human associative learning. In J. A. Wills (Ed.), New directions in human associative learning (pp. 41-64). New Jersey: Whiley.

Jausovec, N. (2000). Differences in cognitive processes between gifted, intelligent, creative, and average individuals while solving complex problems: an EEG study. Intelligence, 28, 213-237. doi: 10.1016/S01602896(00)00037-4.

Jiménez, J. y O'shanahan, I. (2008). Enseñanza de la lectura: de la teoría y la investigación a la práctica educativa. Revista Iberoamericana de Educación. 45, 5-25.

Kane, M.J. y Engle, R. W. (2002). The role of prefrontal cortex in workingmemory capacity, executive attention and general fluid intelligence: And individual-differences perspective. Psychonomic Bulletin and Review, 9, 637671.

Kavanagh, M. y Mohan, Th. (2008). Human Resource Information Systems: Basics, Applications, and Future Directions. Los Angeles: Sage Publications
Lépine, R., Barrouillet, P. y Camos, V. (2005). What makes working memory spans so predictive of high-level cognition? Psychonomic Bulletin y Review, 12, 165-170.

Manzanero, A. (2006). Procesos automáticos y controlados de memoria: Modelo Asociativo (HAM) vs. Sistema de Procesamiento General Abstracto. Revista de Psicologia General y Aplicada, 59, 373-412.

Navarro, J. I., Aguilar, M., Marchena, E., Ruiz, G., Menacho, I. y Van Luit, H. (2011).Longitudinal study of low and high achievers in early mathematics. British Journal of Educational Psychology, 82, 28-41. doi:10.1111/j.2044-8279.2011.02043.x.

Navarro, J. I., Aguilar, M., Alcalde, C., Ruiz, G., Marchena, E. y Menacho, I. (2011). Inhibitory processes, working memory, phonological awareness, naming speed, and early arithmetic achievement. Spanish Journal of Psychology, 14(2), 580-588.

Oberauer, K., Süb, H., Schulze, R., Wilhelm, O. y Wittmann, W. (2000). Working memory capacity-facets of a cognitive ability construct. Personality and Individual Differences, 29, 1017-1046.

Ortiz, R., Jiménez, J. J., Muñetón, M., Rojas, E., Estévez, A., Guzmán, R., Rodríguez, C. y Naranjo, F (2008) Desarrollo de la percepción del habla en niños con dislexia. Psicothema, 20, 678-683.

Pascual-Leone, J. (1978). La teoría de los operadores constructivos. En J. Delval (Ed.), Las teorías, los métodos y el desarrollo temprano (pp. 208-227). Madrid: Alianza.

Pascual-Leone, J. y Johnson, J. M. (2001). Manual for the FIT: Figural intersections test. Toronto, Canadá: Developmental Processes Laboratory, Department of Psychology, York University.

Pérez, C. (2005). Métodos estadísticos avanzados con SPSS. Madrid: Thomson.

Prieto, M.D. y Hervás, R. M. (2001). Estrategias de identificación y asesoramiento de alumnos superdotados. Murcia: Diego Marín.

Proctor, R. W. y Vu K. P. L. (2006). The cognitive revolution at age 50: has the promise of the information processing approach been fulfilled? Journal of Human Computer Interaction, 23, 253-284. doi: 10.1207/s15327590ijhc2103_1.

Raven, J. C. (1994). Standard progresive matrices. Madrid: TEA.

Renzulli, J. R. (1998). The three-ring conceptionof giftedness. In S. M. Baum, S. Reis, y L. R. Maxfields (Eds.), Nurturing the gifts and talents of primary grade students (pp. 123-168). Mansfield Centre, CT: Creative Learning Press.

Sanz de Acedo, M. L. y Sanz de Acedo, M. T. (2008). Instrucciones explícitas para la ejecución creativa según dos tests de creatividad teniendo en cuenta la inteligencia. Anales de Psicología, 24, 129-137.

Sedó, M. (2007). Five Digit Test. Madrid: TEA.

Servera, M. y Llabrés, J. (2004). CSAT. Test de atención sostenida en la infancia. Madrid: TEA.

Schneider, W. y Shiffrin, R. M. (1977). Controlled and automatic human, information processing: Detection, search and attention. Psychological Review, 84, 1-66.

Sternberg, R. J. (1999). The theory of successful intelligence. Review of General Psychology, 3, 292-316.

Wechsler, D. (2005). Escala de Inteligencia de Wechsler para niños. Madrid: TEA.

(Artículo recibido: 25-03-2011, revisado: 31-08-2011, aceptado: 16-06-2012) 